\title{
CORRECTION
}

\section{Correction: RRBP1 rewires cisplatin resistance in oral squamous cell carcinoma by regulating Hippo pathway}

Omprakash Shriwas, Rakesh Arya (D), Sibasish Mohanty, Pallavi Mohapatra, Sugandh Kumar, Rachna Rath, Sandeep Rai Kaushik, Falak Pahwa, Krushna Chandra Murmu, Saroj Kumar Das Majumdar, Dillip Kumar Muduly, Anshuman Dixit, Punit Prasad, Ranjan K. Nanda and Rupesh Dash (D)

British Journal of Cancer (2021) 124:2039; https://doi.org/10.1038/s41416-021-01414-w

Correction to: British Journal of Cancer https://doi.org/10.1038/ s41416-021-01336-7, published online 24 March 2021

The original version of this article unfortunately contained a mistake. During the proof process the authors unfortunately removed of one the co-corresponding authors. The correct version should read: Correspondence: Rupesh Dash (rupesh.dash@gmail. com) or Ranjan K. Nanda (ranjan@icgeb.res.in). The authors apologize for the error. The original article has been corrected. 